OPEN ACCESS

Edited by:

Sally Ann Frautschy, University of California, Los Angeles, United States

Reviewed by: Niccolo Terrando,

Duke University, United States Robert Adam Harris, Karolinska Institutet (KI), Sweden

*Correspondence: Joseph El Khoury jelkhoury@mgh.harvard.edu

Specialty section: This article was submitted to Multiple Sclerosis and

Neuroimmunology,

a section of the journal

Frontiers in Immunology

Received: 15 September 2019 Accepted: 13 November 2019 Published: 02 December 2019

Citation:

Hickman SE, Allison EK, Coleman U, Kingery-Gallagher ND and EI Khoury J (2019) Heterozygous CX3CR1 Deficiency in Microglia Restores Neuronal $\beta$-Amyloid Clearance Pathways and Slows Progression of Alzheimer's Like-Disease in PS1-APP Mice. Front. Immunol. 10:2780 doi: 10.3389/fimmu.2019.02780

\section{Heterozygous CX3CR1 Deficiency in Microglia Restores Neuronal $\beta$-Amyloid Clearance Pathways and Slows Progression of Alzheimer's Like-Disease in PS1-APP Mice}

\author{
Suzanne E. Hickman ${ }^{1}$, Elizabeth K. Allison ${ }^{1}$, Uwanda Coleman ${ }^{1}$, \\ Nathan D. Kingery-Gallagher ${ }^{1}$ and Joseph El Khoury ${ }^{1,2 *}$ \\ ${ }^{1}$ Center for Immunology and Inflammatory Diseases, Massachusetts General Hospital and Harvard Medical School, \\ Charlestown, MA, United States, ${ }^{2}$ Division of Infectious Diseases, Massachusetts General Hospital and Harvard Medical \\ School, Charlestown, MA, United States
}

CX3CR1 is a chemokine receptor expressed on microglia that binds Fractalkine (CX3CL1) and regulates microglial recruitment to sites of neuroinflammation. Full deletion of CX3CR1 in mouse models of Alzheimer's disease have opposing effects on amyloid- $\beta$ and tau pathologies raising concerns about the benefits of targeting CX3CR1 for treatment of this disease. Since most therapies achieve only partial blockade of their targets, we investigated the effects of partial CX3CR1 deficiency on the development and progression of amyloid- $\beta$ deposition in the PS1-APP Alzheimer's mouse model. We generated PS1-APP mice heterozygous for CX3CR1 (PS1-APP-CX3CR $1^{+/-}$) and analyzed these mice for Alzheimer's-like pathology. We found that partial CX3CR1 deficiency was associated with a significant reduction in $A \beta$ levels and in senile-like plaque load in the brain as compared with age-matched PS1-APP mice. Reduced A $\beta$ level in the brain was associated with improved cognitive function. Levels of the neuronal-expressed A $\beta$-degrading enzymes insulysin and matrix metalloproteinase 9, which are reduced in the brains of regular PS1-APP mice, were significantly higher in PS1-APP-CX3CR1 ${ }^{+/-}$ mice. Our data indicate that lowering CX3CR1 levels or partially inhibiting its activity in the brain may be a therapeutic strategy to increase neuronal $A \beta$ clearance, reduce $A \beta$ levels and delay progression of Alzheimer's-Like disease. Our findings also suggest a novel pathway where microglial CX3CR1 can regulates gene expression in neurons.

Keywords: microglia, Alzheimer's disease, CX3CR1, A $\beta$-degrading enzymes, fractalkine, chemokines

\section{INTRODUCTION}

Deposition of amyloid- $\beta(A \beta)$ in the brain is one of the pathological hallmarks of Alzheimer's disease $(A D) . A \beta$ levels in the brain are regulated by several mechanisms: (1) the pathways that generate $A \beta$ consisting of $\beta$-secretase and the $\gamma$-secretase complex (1); (2) the enzymes that clear $\mathrm{A} \beta$ by means of degradation, such as neprilysin, insulysin (IDE), and matrix metalloproteinase 9 (MMP9) (2, 3); (3) clearance of $\mathrm{A} \beta$ through phagocytosis by mononuclear phagocytes/microglia and astrocytes, and (4) transport across the blood brain barrier (4). Progression of Alzheimer's disease is associated with reduced $A \beta$-clearing pathways resulting in $A \beta$ accumulation (4-6). 
Chemokines are chemotactic cytokines that control recruitment of mononuclear phagocytes to sites of inflammation (7). CX3CR1 is a chemokine receptor expressed on microglia and a subset of monocytes $(8,9)$. CX3CR1 and its chemokine ligand fractalkine (CX3CL1) have been implicated in the recruitment of mononuclear phagocytes to sites of inflammation and injury $(7,10,11)$ and play roles in the pathogenesis of several inflammatory conditions, including atherosclerosis $(12,13)$, neuropathic pain (14), and asthma (15). In Alzheimer's disease complete deletion of CX3CR1 in models of amyloid deposition reduced $A \beta$ deposits and enhanced microglial $A \beta$ phagocytic ability $(16,17)$. These studies provided important insight on the role of CX3CR1 in AD and suggested that targeting CX3CR1 activity may be a therapeutic strategy to lower $A \beta$ levels. It is unlikely that pharmacologic targeting of CX3CR1 will achieve the effects of complete deletion of this receptor as in CX3CR1 $1^{-/-}$ mice. However, it is possible to achieve partial inhibition of CX3CR1 activity pharmacologically, similar to what is observed in mice heterozygous for this receptor $\left(\mathrm{CX} 3 \mathrm{CR} 1^{+/-}\right)$.

To investigate the effect of partial CX3CR1 deficiency on progression of $\mathrm{AD}$, we used bigenic mice expressing mutation of presenilin 1 and the amyloid precursor protein with the Swedish mutation (PS1-APP) (18, 19), and analyzed Alzheimer'slike pathology in PS1-APP mice heterozygous for CX3CR1 (PS1APP-CX3CR $1^{+/-}$). We found that partial CX3CR1 deficiency reduced plaque load and $A \beta$ levels in the brains and mitigated the memory deficit in PS1-APP mice. This was associated with increased levels of the neuronal-expressed $A \beta$-degrading enzymes insulysin and MMP9, suggesting that partial microglial CX3CR1 deficiency restores the ability of neurons to clear A $\beta$. Since neuronal fractalkine is the only known ligand for CX3CR1, our results also suggest a novel pathway through which microglia can regulate neuronal gene expression possibly via CX3CR1fractalkine interactions.

\section{MATERIALS AND METHODS}

\section{Mice}

PS1-APP transgenic mice (B6C3-Tg (APPswe, PSEN1dE9)85Dbo/J stock number 004462) were purchased from The Jackson Laboratories and subsequently bred in the animal care facilities at Massachusetts General Hospital to a C57BL6 background. These mice co-express two genetic mutations that are associated with familial AD: a "humanized" Swedish amyloid precursor protein mutation (APP695SWE) and a mutant exon-9-deleted variant of human presenilin 1 (PSEN1/dE9). The APP and PSEN1 transgenes are integrated into a single locus and are independently under the control of separate mouse prion protein promoter elements, which direct expression of the transgenes predominantly to central nervous system neurons $(18,19)$.

CX3CR1-GFP mice were a generous gift from Dan Littman (New York University, New York). In this mouse strain, the CX3CR1 gene has been replaced by Green Fluorescent protein (GFP) reporter gene (20) and is maintained in the animal care facilities at Massachusetts General Hospital on a C57BL6 background. For our experiments, PS1-APP heterozygous mice were crossed with $\mathrm{C} 57 \mathrm{Bl} / 6$ to generate PS1-APP heterozygous progeny and their WT littermates. In parallel breeding, CX3CR1 $1^{-/}$mice were crossed with PS1-APP heterozygous mice and the resulting pups were genotyped at the time of weaning to select PS1-APP-CX3CR $1^{+/-}$genotypes. PS1-APPCX3CR1 ${ }^{+/-} \mathrm{F} 1$ mice were also bred with $\mathrm{CX} 3 \mathrm{CR}^{-/-}$mice to obtain the desired genotypes PS1-APP-CX3CR1 $1^{+/}$, PS1-APPCX3CR1 $1^{-/-}$, WT-CX3CR $1^{+/-}$, and WT-CX3CR1-/-. PS1APP-CX3CR $1^{+/-}$mice are heterozygous for CX3CR1 and PS1APP-CX3CR1 ${ }^{-/-}$mice have no functional CX3CR1 and both genotypes express GFP. In both genotypes, monocytes and microglia appear green under fluorescent microscopy. Because of the limited number of mice that can be generated, only male mice were used at ages ranging from 5 to 24 months and euthanized according to approved institutional procedures. All protocols were approved by the Massachusetts General Hospital Institutional Animal Care and Use Committee and met US National Institutes of Health guidelines for the humane care of animals.

\section{Immunofluorescence Staining for Total $A \beta$-Containing Plaques}

Brains were harvested from transgenic PS1-APP, their WT littermates, PS1-APP-CX3CR1 $1^{-/}$or PS1-APP-CX3CR1 $1^{+/}$and fixed in $2 \%$ paraformaldehyde (PFA) in phosphate buffered saline (PBS) (Mediatech Inc., Manassas, VA) pH 7.5 overnight at $4^{\circ} \mathrm{C}$. The fixed brains were then placed in $30 \%$ sucrose overnight at $4^{\circ} \mathrm{C}$ for cryoprotection. Brains were embedded in TissueTek O.C.T compound (Sakura Finetek USA Inc., Torrance, CA) and cut into $10-12 \mu \mathrm{m}$ frozen sections. To stain plaques of all sizes, sections were blocked with PBS/0.3\%Triton-X 100/2\% goat serum for $30 \mathrm{~min}$, then incubated at $25^{\circ} \mathrm{C}$ overnight with rabbit anti-A $\beta$ pan antibody (Life Technologies, Grand Island, $\mathrm{NY}$ ) in $0.3 \%$ Triton $\mathrm{X}-100$ and $2 \%$ goat serum. The slides were rinsed three times in PBS/0.3\% TritonX-100 and then incubated with anti-rabbit IgG coupled to Alexa ${ }^{555}$, rinsed and mounted with VectaShield (Vector Laboratories Inc., Burlingame, CA). Slides were viewed by fluorescence microscopy and digitally photographed on Nikon Eclipse ME600 microscope with Nikon DXM 1200C camera system at $40 \mathrm{X}$ total magnification. The number of $\mathrm{A} \beta$ deposits in the cortex and hippocampus was quantified using Nikon NIS Elements Advanced Research Software, version 2.3. The number of $\mathrm{A} \beta$ deposits stained with the antibody was measured for each slice using three slices per mouse. $A \beta$-deposit area fraction $(\mathrm{A} \beta \mathrm{AF})$, which is the total area stained for $A \beta$ counted in a defined region relative to the total area of that region, was calculated by the software.

\section{Staining for Insulysin and Other Cell Markers}

Frozen sections were fixed in acetone for 2 min then blocked for $1 \mathrm{~h}$ with $1.5 \%$ donkey serum in PBS. Sections were then costained for insulysin with goat anti-IDE $(1 \mu \mathrm{g} / \mathrm{ml}$, Sigma $)$ and for the astrocyte marker GFAP with rabbit anti-GFAP $(5 \mu \mathrm{g} / \mathrm{ml}$, DAKO North America Inc., Carpinteria, CA) or for insulysin and a neuronal marker MAP2 (1:1,000, Abcam, Cambridge, MA). 
Control antibodies from the same companies as the primary antibodies were used in parallel. After rinsing three times in PBS secondary antibodies (Life Technologies) were added for $30 \mathrm{~min}$ : Alexa ${ }^{488}$-labeled donkey anti-rabbit $(4 \mu \mathrm{g} / \mathrm{ml})$, Alexa ${ }^{555}$-labeled donkey anti-goat $(4 \mu \mathrm{g} / \mathrm{ml})$. Slides were then rinsed and mounted with VectaMount and digitally photographed via fluorescence microscopy at total magnification of 400X.

\section{Isolation of CD11b+ Cells}

Transgenic PS1-APP, their WT litter mates, PS1APP/CX3CR $1^{-/-}$or PS1-APP/CX3CR1 $1^{+/-}$were euthanized and perfused with $30 \mathrm{cc}$ PBS without $\mathrm{Ca}^{++}$and $\mathrm{Mg}^{++}$ $\left(\mathrm{PBS}^{=}\right)$. Brains were then removed, rinsed in $\mathrm{PBS}^{=}$and placed separately into a C-tube (Miltenyi Biotech, Auburn, CA) with RPMI (no phenol red) containing $2 \mathrm{mM}$ L-glutamine (Mediatech), Dispase $(2 \mathrm{U} / \mathrm{ml})$ and $0.2 \%$ Collagenase Type 3 (Worthington Biochemicals, Lakewood, NJ) according to our previous protocol (5). Brains were processed using the gentleMACS Dissociator (Miltenyi Biotech) on the brain program settings according to manufacturer's directions. Briefly, the brains were subjected to three rounds of dissociation each followed by a period of incubation at $37^{\circ} \mathrm{C}$. After the second round of dissociation, DNase I grade II (Roche Applied Science, Indianapolis, IN) was added at a concentration of $40 \mathrm{U} / \mathrm{ml}$ and incubated for an additional $10 \mathrm{~min}$ before the final round of dissociation. The digestion enzymes were inactivated by addition of $\mathrm{PBS}=$ containing $2 \mathrm{mM}$ EDTA and $5 \%$ fetal bovine serum (FBS) and the digested brain bits were triturated gently and passed over a $100 \mu \mathrm{m}$ filter (Fisher Scientific, Pittsburgh, PA). Cell pellets were resuspended in $10.5 \mathrm{ml} \mathrm{RPMI} / \mathrm{L}$ glutamine, mixed gently with $4.5 \mathrm{ml}$ physiologic Percoll ${ }^{\circledR}$ (Sigma Aldrich), and centrifuged at $850 \times \mathrm{g}$ for $45 \mathrm{~min}$. The cell pellet was resuspended in $\mathrm{PBS}^{=}$and then passed over a $40 \mu \mathrm{m}$ filter (Fisher Scientific), centrifuged and washed again with $\mathrm{PBS}^{=}$. Microglia were then isolated as previously described (9). The number of cells isolated per hemisphere were recorded as shown in Figure 2C. Cells were centrifuged, and the pellets were lysed in RLT-Plus buffer from the RNeasy ${ }^{\circledR}$ Plus mini kit (Qiagen Inc., Valencia, CA) to use for QPCR.

\section{Co-staining of Brains for CD11b and $A \beta$ Plaques}

Frozen sections from PS1-APP, WT littermates, and PS1$\mathrm{APP} / \mathrm{CX} 3 \mathrm{CR} 1^{-/}$or PS1-APP/CX3CR $1^{+/-}$were fixed in acetone for $2 \mathrm{~min}$, washed in PBS, then treated with $0.25 \%$ trypsin for antigen retrieval. Endogenous peroxidase activity was quenched with $0.3 \% \mathrm{H}_{2} \mathrm{O}_{2}$ followed by blocking with $1.5 \%$ donkey serum in PBS. Sections were incubated overnight at $25^{\circ} \mathrm{C}$ with rat anti-CD11b (clone 5C6) (AbD Serotec) or rat IgG2b negative control (AbD Serotec) each at $10 \mu \mathrm{g} / \mathrm{ml}$ in PBS with $1.5 \%$ donkey serum. The slides were then processed using the Vectastain ${ }^{\circledR}$ Elite $\mathrm{ABC}$ reagent (Vector laboratories) according to the manufacturer's instructions followed by development with the NovaRed ${ }^{\mathrm{TM}}$ Peroxidase Substrate kit. $\mathrm{A} \beta$-containing plaques were stained with 1\% Thioflavin-S (Sigma Aldrich) for $5 \mathrm{~min}$ in the dark.
Finally, the sections were counterstained with hematoxylin, mounted with VectaMount and digitally photographed via brightfield microscopy to detect CD11b, and via fluorescence to visualize Thioflavin-S. Since different size plaques can exist within any single mouse at any single age, the size of the plaques was determined using Image $J$ and the number of CD11b positive cells associated with plaques $\geq 75 \mu \mathrm{m}$ in their largest diameter was quantified by two independent blinded laboratory members. A minimum of 80 plaques were counted per genotype.

\section{Quantification of $A \beta(1-42)$ and $A \beta(1-40)$}

Brains from transgenic PS1-APP, WT littermates, and PS1APP-CX3CR1 $1^{+/}$were harvested sans cerebellum, weighed and homogenized in $8 \mathrm{X}$ weight/volume of $5 \mathrm{M}$ guanidine using the TissueRuptor (Qiagen) homogenizer. Total $\mathrm{A} \beta$ (1$42)$ and $A \beta$ (1-40) were measured in whole brain homogenates using colorimetric immunoassay kit for human $A \beta$ (1-42) and $A \beta$ (1-40) (Life Technologies) according to manufacturer's instructions. Briefly, brain homogenates were diluted and added to wells coated with a monoclonal antibody specific for the NH-terminus of human $\mathrm{A} \beta$ and co-incubated with a rabbit antibody specific for the $\mathrm{COOH}$-terminus of $\mathrm{A} \beta$ (1$42)$ or $A \beta(1-40)$. Horseradish peroxidase labeled anti-rabbit IgG is used to detect bound rabbit antibody through use of substrate solution. Amount of $A \beta(1-42)$ and $A \beta$ (1-40) in brain samples was determine using a standard curve of known amounts of human $A \beta$ (1-42) or $A \beta$ (1-40) supplied with the kits.

\section{Quantitative Real Time PCR}

Total RNA from CD11b + cells was isolated using the RNeasy ${ }^{\circledR}$ Plus mini kit for RNA isolation (Qiagen) and RNA from whole brain homogenates was isolated using the RNeasy ${ }^{\circledR}$ Lipid Tissue kit (Qiagen) according to the manufacturer's instructions and quantified using the Nanodrop ${ }^{\mathrm{TM}} 2000$ (Thermo Fisher Scientific, Waltham, MA). RNAs (500 $\mathrm{ng}-1.5 \mu \mathrm{g}$ ) were reverse transcribed using the $\mathrm{RT}^{2-}$ first strand kit (SA Biosciences). The $\mathrm{qPCR}$ was performed with the MX4000 ${ }^{\mathrm{TM}}$ unit (Agilent Technologies, Santa Clara, CA) using SYBR Green to detect the amplification products as described $(21,22)$. The following cycles were performed: initial denaturation cycle $95^{\circ} \mathrm{C}$ for $10 \mathrm{~min}$, followed by 40 amplification cycles of $95^{\circ} \mathrm{C}$ for $15 \mathrm{~s}$ and $60^{\circ} \mathrm{C}$ for $1 \mathrm{~min}$ and ending with one cycle at $25^{\circ} \mathrm{C}$ for $15 \mathrm{~s}$. Analysis was performed on the data output from the MX4000 ${ }^{\mathrm{TM}}$ software (Agilent technologies) using Microsoft Excel XP. Relative quantification of mRNA expression was calculated by the comparative cycle method described by the manufacturer (Agilent technologies). Primer sequences used were as follows: CX3CR1 (forward) ACCGGTACCT TGCCATCGT (reverse) ACACCGTGCTGCACTGTCC. $\beta 2$ microglobulin (forward) CCGAACATACTGAACTGCTAC (Reverse) CCCGTTCTTCAGCATTTGGA. Insulysin (Forward) GAAGACAAACGGGAATACCGTG (Reverse) CCGCTG AGGACTTGTCTGTG. Neprilysin (Forward) GCAGC CTCAGCCGAAACTAC (Reverse) CACCGTCTCCATGTT 
GCAGT. MMP9 (Forward) GCCATGCACTGGGCTTAGAT (Reverse) TCTTTATTCAGAGGGAAGCCCTC.

\section{Behavioral Studies: Barnes Maze}

The Barnes maze is a spatial learning task that allows a subject to escape from aversive stimuli, such as bright light, by using environmental cues to locate an escape box. The Barnes maze (Stoelting, Wood Dale, IL) is a light gray circular acrylic table top that is $91 \mathrm{~cm}$ in diameter with 20 equally spaced holes that are each $5 \mathrm{~cm}$ in diameter and $5 \mathrm{~cm}$ from the edge. Under one of the holes an escape box (target box) is mounted that allows an animal to enter and escape while the remaining holes are equipped with false escape boxes that do not allow entry but will remove any cues that may be viewed through open holes. We used a protocol that allowed detection of spatial learning deficits in PS1-APP mice that had been trained first with the cued version followed by the non-cued version (23). Mice show impaired spatial learning in the non-cued, hidden-fixed target version, but not in the cued-variable version. In the cued-variable version of the Barnes maze, the target hole is marked with a conspicuous polystyrene cone next to the target hole on the maze surface and the target location is varied from trial to trial. In the non-cued, hidden target version, the target hole was always located in the same place relative to extra-maze cues, but the maze itself was rotated between trials and thoroughly cleaned to eliminate intra-maze cues such as odor. Using this protocol, we tested WT, PS1-APP, and PS1-APP/CX3CR1-/+ mice 280-300 days of age. To begin each trial, the subject was placed in the middle of the maze and covered with a black box for $10 \mathrm{~s}$ before being released. The mouse was then allowed to explore the maze until it entered the target box up to $180 \mathrm{~s}$. If a mouse did not find the target after $180 \mathrm{~s}$, it was gently guided to the correct hole and allowed to enter. Once in the escape box, the hole was covered, and the mouse was allowed to sit inside the box for $1 \mathrm{~min}$ before being gently returned to its home cage. Training sessions comprised three trials per mouse, with a rest interval of 20-25 min between trials. On the fifth day, a single 90-s probe trial was run in which all holes were blocked and the number of pokes into the "target" hole and adjacent holes were counted. The probe trial is performed in order to determine if the mouse remembers the location of the target. Cued-variable target and non-cued, hidden target sessions were run on four consecutive days each. The principle measure was errors time to enter target hole (escape latency). All experiments were videotaped, and the input was analyzed using the AnyMaze software (Stoelting).

\section{Months}

\section{Pan $A \beta$ Immunostaining}
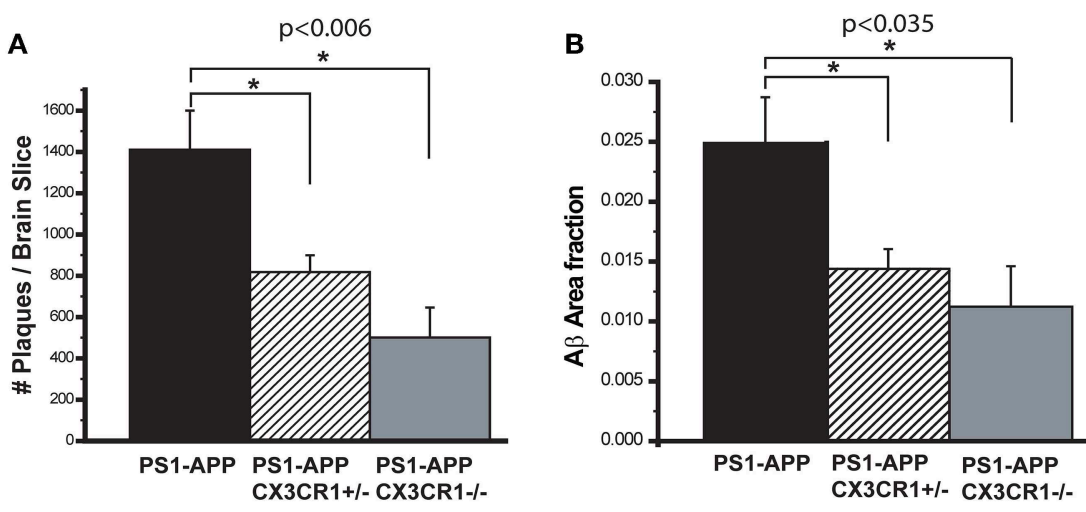

D
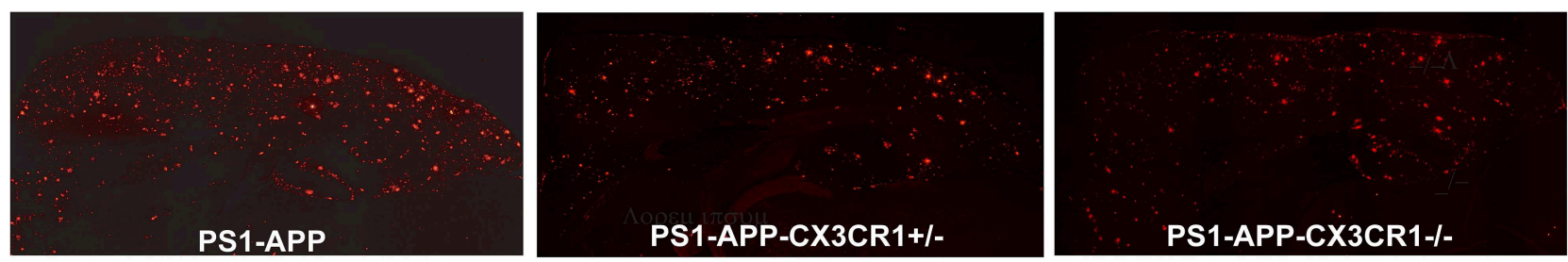

FIGURE 1 | CX3CR1 deficiency results in reduced A $\beta$ deposits and levels of A $\beta$ in 10-month old PS1-APP-CX3CR1-deficient genotypes. (A-C) Fixed brain sections from 10-month old PS1-APP, PS1-APP-CX3CR1 $1^{+/-}$, and PS1-APP-CX3CR1 $1^{-/-}$mice were stained using polyclonal pan-A $\beta$ antibody. The number of A $\beta$ deposits (A) and $A \beta$-deposit area fraction (B) were determined using three sections per mouse (Values represent group mean \pm SEM). (C) Quantitative PCR measurements of CX3CR1 RNA in 10-month old PS1-APP, PS1-APP-CX3CR1 ${ }^{+/-}$, and PS1-APP-CX3CR1 $1^{-/-}$microglia show a gene dosage effect. (D) Representative fluorescent micrographs of plaques (red) in PS1-APP, PS1-APP-CX3CR1 ${ }^{+/-}$, and PS1-APP-CX3CR1 ${ }^{-/-}$mice. For all measurements in this figure, $n=6$ for PS1-APP, $n=7$ for PS1-APP-CX3CR1 ${ }^{+/-}$, and $n=3$ for PS1-APP-CX3CR $1^{-/-}$. * represent statistically significant. 


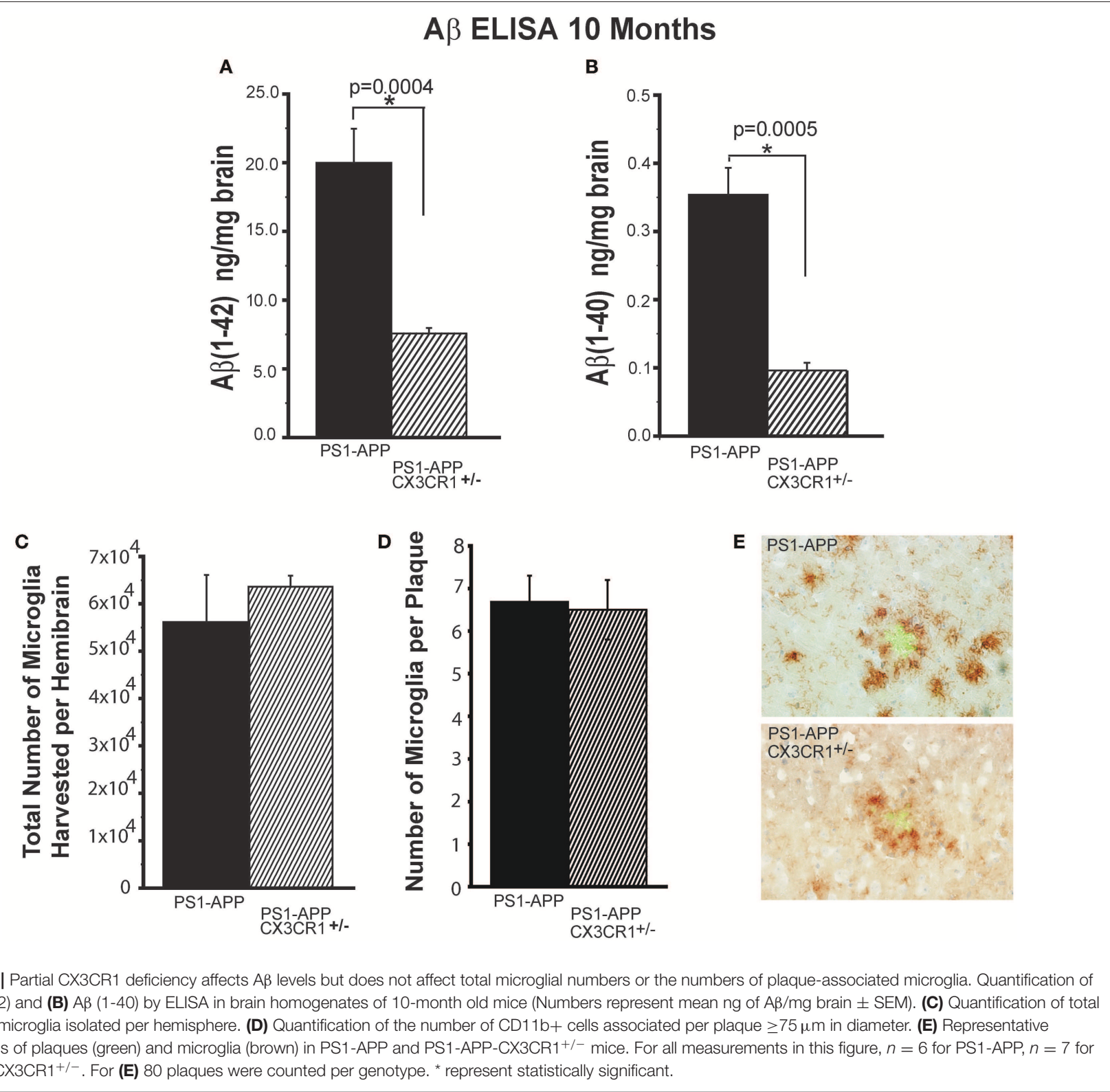

\section{Statistical Analysis}

Statistical analysis was performed using student $T$-test or oneway ANOVA with between-groups differences determined by Tukey analysis provided in the "Microcal Origin 8" graphics and statistics software and $p<0.05$ were considered significant.

\section{RESULTS}

\section{CX3CR1 Deficiency Is Associated With Reduced Plaque Load and Reduced A $\beta$ Levels in PS1-APP Mice}

To determine the effect of CX3CR1 deficiency on AD-like pathology in PS1-APP mice, we assessed A $\beta$ load and levels in brains of PS1-APP transgenic mice and PS1-APP-CX3CR1 deficient mice at 10 months of age. In studies to assess $A \beta$ load, fixed-frozen sections were stained with a polyclonal pan- $A \beta$ antibody that recognizes both $A \beta(1-42)$ and $A \beta$ (1-40) in areas of $A \beta$ deposit. The number of $A \beta$ deposits per section (cortex + hippocampus) and $A \beta$-deposit area fraction $(\mathrm{A} \beta \mathrm{AF})$ were quantified in PS1-APP, PS1-APP-CX3CR1 ${ }^{+/-}$, and PS1-APPCX3CR1 ${ }^{-/-}$mice. Compared with PS1-APP mice, there were significant decreases in the number of $A \beta$-deposits (Figure 1A) and A $\beta A F$ (Figure 1B) in both PS1-APP-CX3CR1-deficient genotypes. The number of $\mathrm{A} \beta$ deposits per section in PS1APP-CX3CR $1^{+/-}$and APP-CX3CR $1^{-/-}$mice was reduced to $58 \%(818 \pm 81, p=0.006)$ and $35 \%(501 \pm 144, p=$ $0.007)$, respectively, of PS1-APP mice (1,409 \pm 189$)$. PS1APP-CX3CR $1^{-/}$mice did not have a statistically different number of $\mathrm{A} \beta$ deposits than PS1-APP-CX3CR1 ${ }^{+/-}$mice. 
A

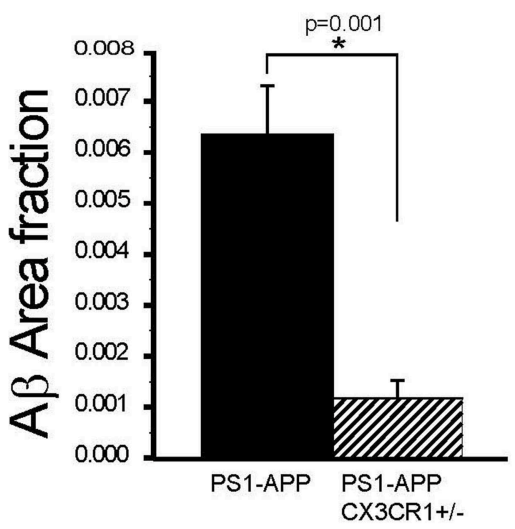

B 24 Months

FIGURE 3 | CX3CR1 deficiency results in reduced plaque-load in 5- and 24-month old PS1-APP-CX3CR1+/- mice. (A,B) Brain sections from PS1-APP and PS1-APP-CX3CR $1^{+/-}$mice at 5 and 24-months of age were stained and A 3 area fraction (ABAF) was determined for hippocampus + cortex in mice at 5 and 24-months of age. (A) At 5 months of age ABAF is significantly reduced in PS1-APP-CX3CR1 $+/-(n=3)$ mice compared with PS1-APP mice $(n=8)$. (B) At 24months of age, ABAF is also significantly reduced in PS1-APP-CX3CR1 ${ }^{+/-}(n=5)$ mice compared with PS1-APP mice $(n=6)$. For all groups three sections per mouse were quantified and numbers represent group mean \pm SEM. * represent statistically significant.

In addition to reduced $\mathrm{A} \beta$-deposit number, the $\mathrm{A} \beta \mathrm{AF}$ was also significantly reduced in PS1-APP-CX3CR1-deficient mice compared to PS1-APP mice. A $3 A F$ in PS1-APP-CX3CR $1^{+/-}$ mice was reduced to $60 \%$ of PS1-APP values $(0.015 \pm 0.0017$ vs. $0.025 \pm 0.0038, p=0.035)$ and in PS1-APP-CX3CR $1^{-/-}$ mice it was reduced to $44.8 \%(0.0112 \pm 0.0033$ vs. 0.025 $\pm 0.0038, p=0.023)$. The difference in A $\beta$ AF of PS1-APPCX3CR $1^{+/-}$and PS1-APP-CX3CR $1^{-/-}$was not statistically significant. Quantitative PCR analysis confirmed that CX3CR1 RNA was not detectable in microglia isolated from PS1APP-CX3CR1 ${ }^{-/-}$mice and that level of CX3CR1 RNA in microglia from PS1-APP-CX3CR $1^{+/-}$mice was $\sim 54 \%$ of that of microglia from PS1-APP mice confirming a gene dosage effect on CX3CR1 expression (Figure 1C). Figure 1D shows examples of immunofluorescent staining with Pan-A $\beta$ antibody in PS1-APP, PS1-APP-CX3CR1 $1^{+/-}$, and PS1-APP-CX3CR1 $1^{-/-}$brains.

Because there were no significant differences in $A \beta$ deposits between PS1-APP-CX3CR $1^{+/-}$and PS1-APP-CX3CR $1^{-/}$, we focused subsequent analysis on PS1-APP-CX3CR1 $1^{+/-}$mice. The reduction in plaque number and $\mathrm{A} \beta \mathrm{AF}$ in PS1-APP-CX3CR1deficient mice correlated with reduced levels of $A \beta$ (1-42) and $\mathrm{A} \beta$ (1-40) in brain homogenates as determined by a commercial ELISA kit (Figures 2A,B). There was a 2.6-fold decrease in $A \beta$ (1-42) in PS1-APP-CX3CR $1^{+/-}$mice compared with PS1-APP mice (Figure 2A) $(7.56 \mathrm{ng} / \mathrm{mg}$ brain \pm 0.41 vs. $19.8 \pm 2.6 \mathrm{ng} / \mathrm{mg}$ brain, $p=0.0004$. Levels of $\mathrm{A} \beta$ (1-40) were also significantly reduced in PS1-APP-CX3CR $1^{+/-}$mice (Figure $2 \mathrm{~B}$ ). A $\beta$ (140) levels were 3.5-fold lower in PS1-APP-CX3CR ${ }^{+/-}$brain homogenates compared with PS1-APP brains $(0.10 \pm 0.011$ vs. $0.35 \pm 0.039 \mathrm{ng} / \mathrm{mg}$ brain, $p=0.0005)$. These data indicate that partial CX3CR1 deficiency in heterozygous PS1-APP-CX3CR ${ }^{+/-}$ is associated with a significant reduction in $A \beta$ deposits and load and in the levels of $A \beta$ (1-42) and (1-40) compared to
PS1-APP mice and that complete deletion of CX3CR1 is not necessary to confer beneficial effects on AD-like pathology in these mice.

\section{Partial CX3CR1 Deficiency Does Not Affect Microglial Number and Association With Plaques of the Same Size}

To determine if partial CX3CR1 deficiency affects overall microglial numbers in the brain, we quantified the total number of microglia harvested per hemisphere of PS1-APP-CX3CR1 $1^{+/-}$ and PS1-APP mice and found no statistically significant differences between the two genotypes (Figure 2C). We also compared the numbers of CD11b + cells associated per plaque and found that when comparing same size plaques $(\geq 75 \mu \mathrm{m})$ there were no statistically significant differences between the two genotypes (Figures 2D,E). These data show that partial CX3CR1 deficiency does not affect the total number of microglia or accumulation of microglia at sites of $\mathrm{A} \beta$ accumulation.

\section{Partial CX3CR1 Deficiency in PS1-APP-CX3CR1+/- Mice Is Associated With Reduced $A \beta$ Load as Early as 5 Months of Age and Continues to 24 Months} To determine if the effect of partial CX3CR1 deficiency on $\mathrm{A} \beta$ deposition and levels starts early and persists at advanced stages of disease in PS1-APP mice, we assessed the effect of CX3CR1 deficiency on A $\beta A F$ in brains of PS1-APP and PS1$\mathrm{APP} / \mathrm{CX} 3 \mathrm{CR} 1^{+/-}$mice at 5 and 24 months of age. In 5month old animals there was a significant 5.5-fold reduction in $\mathrm{A} \beta \mathrm{AF}$ (Figure 3A) in PS1-APP-CX3CR $1^{+/-}$compared with PS1-APP mice ( $0.0011 \pm 0.0003$ vs. $0.0061 \pm 0.0009$, respectively, $p=0.001)$. 


\section{Expression of Insulysin RNA in Whole Brain}

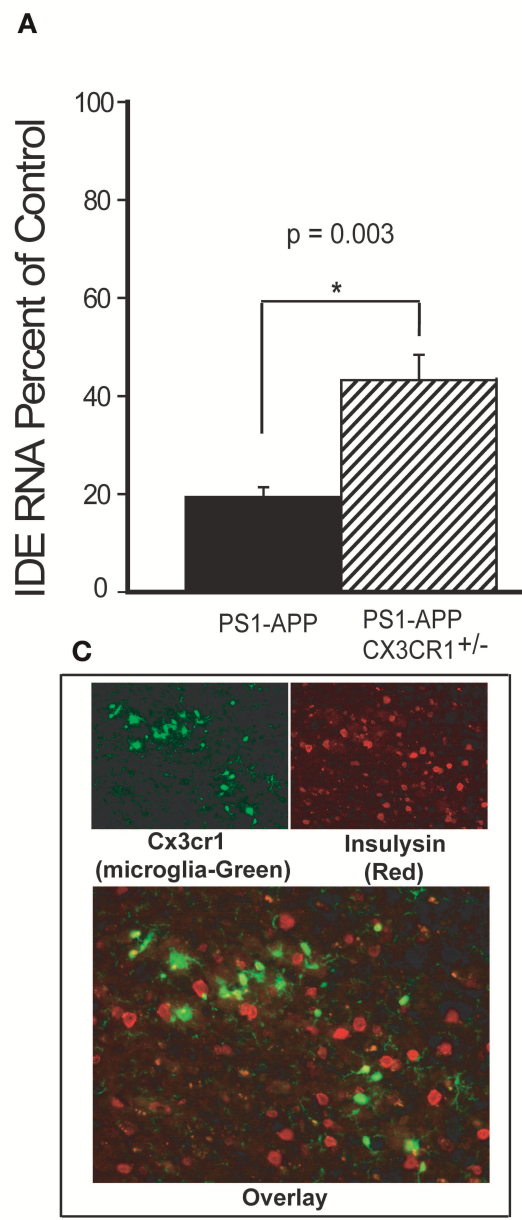

B
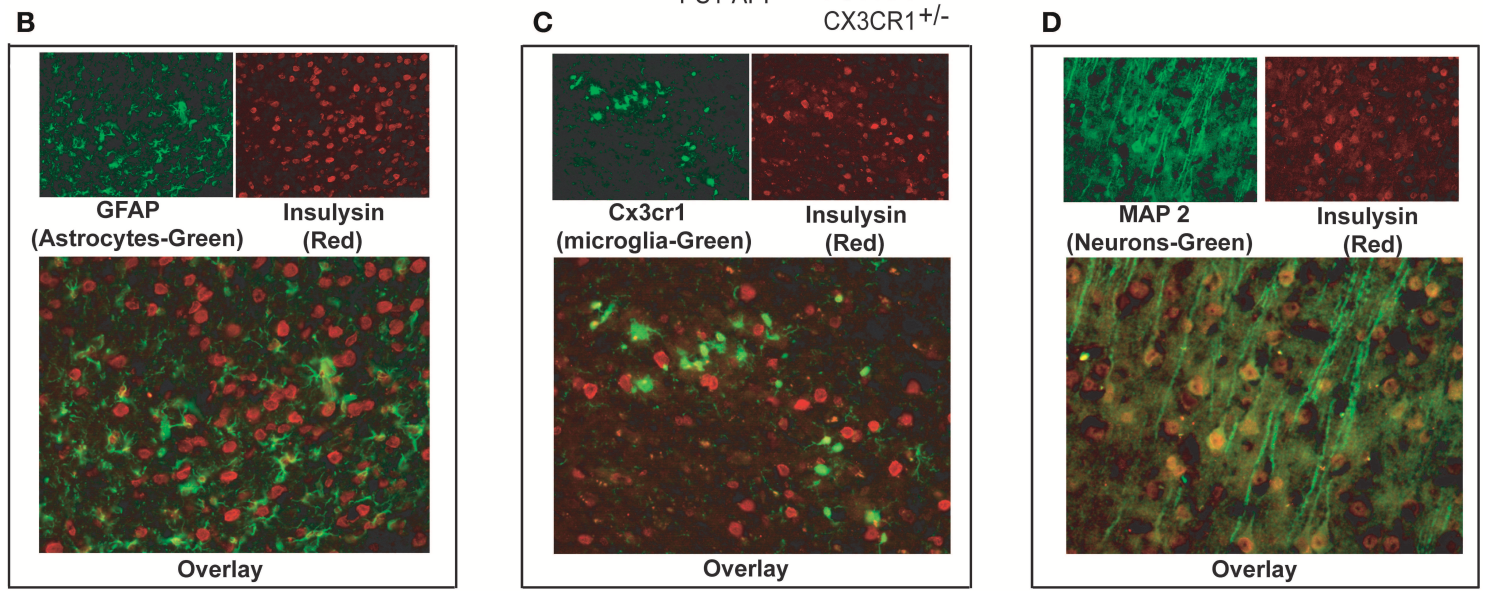

FIGURE 4 | Increased levels of insulysin RNA in brains of CX3CR1-deficient PS1-APP transgenic mice. (A) RNA was extracted from brains of PS1-APP, WT, PS1-APP-CX3CR $1^{+/-}$, and CX3CR $1^{+/-}$mice and assessed for expression of insulysin (IDE) by GPCR. Data represent mean percent of appropriate control \pm SEM ( $n=6$ per genotype). (B-D) CX3CR1-dficient or WT mouse brain sections were co-stained for IDE and cell-specific markers and photographed by fluorescence microscopy at 40X magnification. (B) WT brain section co-stained for the astrocyte marker GFAP (green cells), for IDE (red cells). Most of the cells stained for IDE do not express GFAP. (C) CX3CR1-deficient brain section showing GFP-expressing microglia (green) and stained for IDE (red cells). Most of the microglia do not show detectable levels of IDE. (D) WT mouse brain slices co-stained for a neuronal marker MAP2 (green cells) and IDE (red cells). Most of the MAP2-expressing cells (neurons) also express IDE. * represent statistically significant.

Similarly, at 24-months of age there were significant decreases in $\mathrm{A} \beta \mathrm{AF}$ in $-\mathrm{APP}-\mathrm{CX} 3 \mathrm{CR} 1^{+/-}(38 \%$ reduction, $0.027 \pm 0.003$, $p=0.02)$ compared with PS1-APP brains $(0.044 \pm 0.0034)$ (Figure 3B). These data indicate that partial CX3CR1 deficiency slows the accumulation of A $\beta$ in PS1-APP mice starting early in the disease process and continues to do so at late disease stages.

\section{CX3CR1 Deficiency Is Associated With Increased IDE Levels in PS1-APP-CX3CR1+/- Mice}

$\mathrm{A} \beta$ levels in the brain are regulated in part by several $A \beta$ degrading enzymes, including insulin degrading enzyme (IDEinsulysin), neprilysin and matrix metalloprotease 9 (MMP9) (4).
Progression of Alzheimer's disease in humans and mouse models of the disease is associated with reduced expression of these $\mathrm{A} \beta$-clearing enzymes resulting in decreased $\mathrm{A} \beta$ clearance and increased $\mathrm{A} \beta$ accumulation $(5,24)$. Because partial CX3CR1 deficiency is associated with lower $A \beta$ levels and decreased plaque load in PS1-APP mice, we hypothesized that under pathological conditions such as $\mathrm{AD}, \mathrm{CX} 3 \mathrm{CR} 1$ may regulate expression of $\mathrm{A} \beta$ degrading enzymes. To test this hypothesis, we measured RNA levels of IDE, neprilysin, and MMP9 in brain homogenates of PS1-APP, their wild type (WT) littermate controls, PS1APPCX3CR $1^{+/-}$mice and their CX3CR $1^{+/-}$littermate controls. IDE RNA (expressed as percent of appropriate littermate controls), was reduced in brains of PS1APP to $19.0 \%( \pm 2.0)$ of control level while in PS1-APP-CX3CR $1^{+/-}$it was reduced 


\section{Expression of MMP9 RNA in Brain}

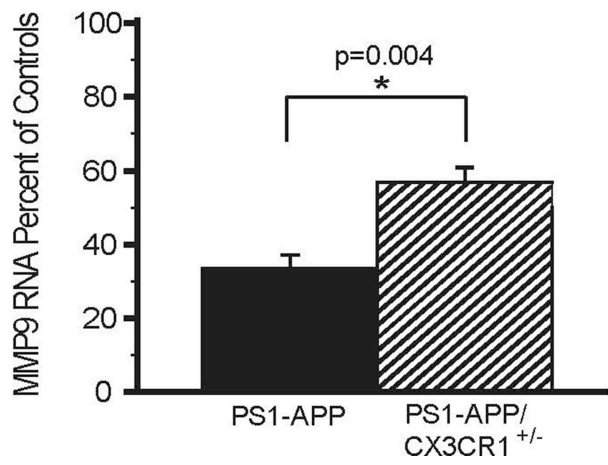

FIGURE 5 | Effects of CX3CR1-deficiency on levels of MMP9 RNA in the whole brains of PS1-APP mice. RNA was extracted from brains of PS1-APP, WT, PS1-APP-CX3CR $1^{+/-}$, and CX3CR $1^{+/-}$mice and assessed for expression of MMP9 by qPCR. Data are expressed as mean percent of appropriate control \pm SEM $\left(n=6\right.$ per genotype). ${ }^{*}$ represent statistically significant.

to only $43 \%( \pm 5.1)$ of control (Figure $4 \mathrm{~A})$. The differences in expression of IDE RNA between PS1-APP mice and PS1APP-CX3CR $1^{+/-}$mice was significant $(p=0.003)$. These data indicate that there is an inverse relationship between CX3CR1 and IDE expression and suggest that partial CX3CR1 deficiency upregulates expression of IDE.

To identify the brain cells that predominantly expressed IDE, fixed frozen sections from CX3CR1deficient brains were stained for IDE and sections from WT mice were co-stained for IDE and MAP2 (neuronal marker) or for IDE and GFAP (astrocyte marker). Co-staining for IDE (red) and GFAP (green, Figure 4B) showed very little co-staining of IDE in GFAP containing cells and intense staining for IDE in cells lacking GFAP. In Figure 4C, GFP-positive microglia are seen evenly distributed or as small clusters, but IDE was much less detectable in microglia compared with intense positive staining seen in other cells throughout the brain. In contrast, co-staining for IDE (red) and MAP2 (green) shows intense positive stain for IDE in neurons containing MAP2 (yellow, Figure 4D). It is possible that detection of IDE protein in microglia and astrocytes is limited by the sensitivity of the staining assay. However, our data strongly suggests that the bulk of IDE expression in the brain occurs in neurons.

\section{Effect of CX3CR1 Deficiency on Levels of Neprilysin and MMP9 in Brains of PS1-APP/CX3CR1-Deficient Mice}

In addition to IDE, $A \beta$ levels in the brain can be regulated by neprilysin, another important $A \beta$-degrading enzyme. To determine if CX3CR1 deficiency is also associated with upregulation of neprilysin levels similar to what we observed with IDE, we measured expression of neprilysin in brains of PS1APP, their WT littermate controls, PS1APP-CX3CR $1^{+/-}$mice, and their controls in the brain. In contrast to what we observed with IDE, we did not find any significant differences in neprilysin expression in the brains of PS1-APP and PS1-APP/CX3CR $1^{+/-}$ mice (Data not shown).

MMP9 is an important $A \beta$-degrading enzyme expressed on microglia (25) and neurons (26). To determine if CX3CR1 deficiency is also associated with up-regulation of MMP9 levels similar to what we observed with IDE, we measured MMP9 RNA in the brains of PS1-APP, PS1APP-CX3CR1 $1^{+/-}$, and their CX3CR $1^{+/-}$littermates. There were statistically significant differences in MMP9 expression in PS1-APP-CX3CR1 ${ }^{+/-}$ compared with PS1-APP mice (Figure 5). PS1-APP mice MMP9 levels fell to $33.6 \%( \pm 3.5)$ of controls, while PS1APP/CX3CR $1^{+/-}$mice MMP9 expression was only reduced to $56.9 \%( \pm 3.9)$ of controls. Compared with PS1-APP mice, MMP9 levels in PS1-APP/CX3CR1 $1^{+/-}$were $69 \%$ higher $(p=$ 0.004). These data indicate that, similar to IDE, partial CX3CR1deficiency mitigates the reduction in MMP9 observed in aging PS1-APP mice.

\section{CX3CR1 Deficiency Is Associated With Increased Levels of $A \beta$-Degrading Enzymes in Brain Homogenates of PS1-APP-CX3CR1 ${ }^{+/-}$Mice but Not in Their Microglia \\ We have previously shown that expression of IDE in microglia} from PS1-APP mice decreases with age and progression of ADlike pathology (5). To determine if the significant preservation of IDE in PS1-APP-CX3CR1-deficient mice occurs in microglia as well as in whole brain, we measured IDE (Figure 6A) and MMP9 (Figure 6B) expression in freshly isolated microglia from 24-month old WT, PS1-APP, and PS1-APP-CX3CR1 ${ }^{+/-}$ mice. IDE RNA levels in PS1-APP microglia were significantly reduced to $42 \%$ of WT control microglia $(0.0056 \pm 0.00055$ vs. $0.014 \pm 0.002, p=0.0003)$. IDE expression in PS1APP/CX3CR $1^{+/-}$microglia was also significantly decreased to $45 \%$ of WT control levels $(0.0063 \pm 0.00039$ vs. $0.014 \pm 0.002$, $p=0.001$ ). In contrast to findings in whole brain where IDE levels in PS1-APP/CX3CR1 $1^{+/-}$mice were significantly higher than in PS1-APP mice, IDE expression in microglia from PS1APP/CX3CR $1^{+/-}$remained at the lower levels seen in PS1-APP mice. These data indicate that CX3CR1 deficiency in microglia results in preservation or maintenance of IDE levels in brain cells other than microglia. IDE expression in microglia from WT mice was 54-fold lower than in whole brains from mice of the same age, suggesting that the bulk of IDE expressed in the brain of 2-year old mice occurs in cells other than microglia.

In contrast, expression of MMP9 in WT microglia was 6fold higher than in whole brain $(0.0062 \pm 0.0018$ vs. $0.00097 \pm$ 0.00015 , respectively) indicating that MMP9 is highly expressed in microglia but is also expressed in other cells of the brain. Expression of MMP9 in microglia from PS1-APP mice and PS1-APP/CX3CR $1^{+/-}$mice was significantly decreased to $23 \%$ and $20 \%$ of WT microglia $(0.0014 \pm 0.00029$ and $0.0012 \pm$ 0.00029 vs. $0.006 \pm 0.0019$ ), respectively. These data indicate that microglial CX3CR1 deficiency did not preserve expression 


\section{Expression of $A \beta$-degrading Enzymes in Microglia}
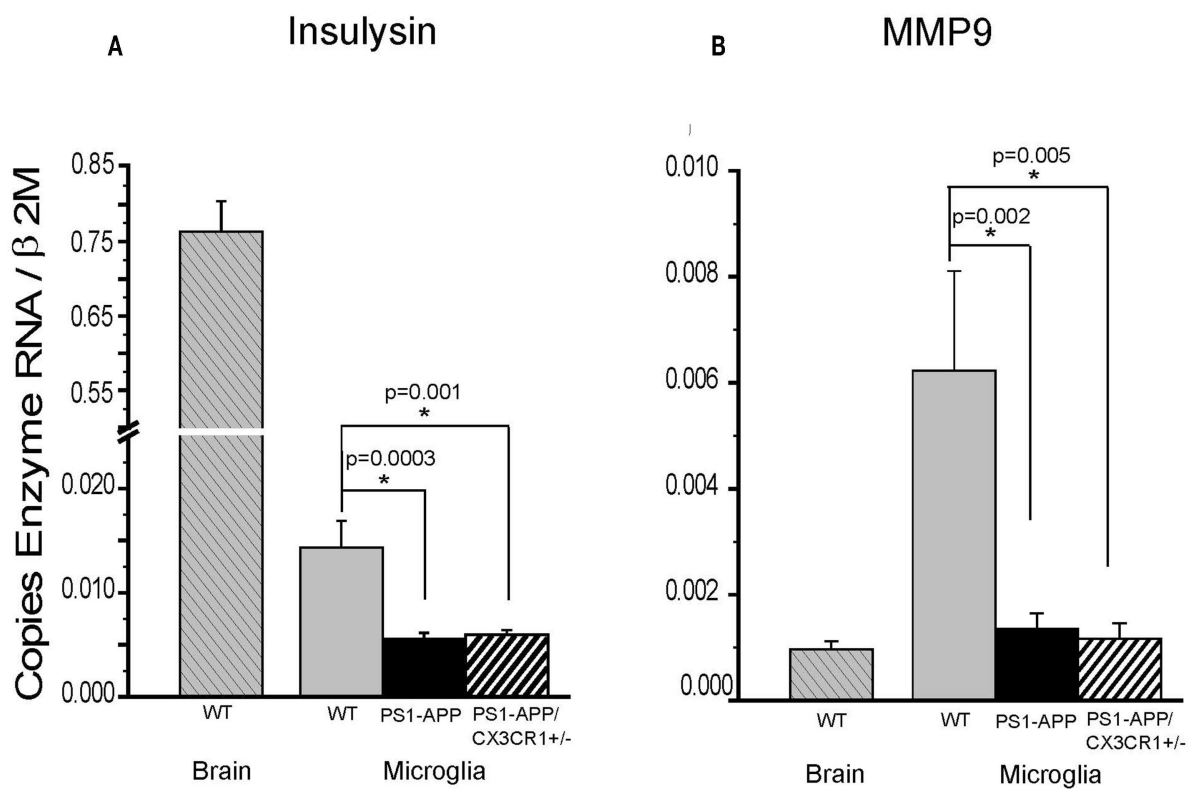

FIGURE 6 | Effects of CX3CR1 deficiency on expression of IDE and MMP9 in microglia from WT, PS1-APP, and PS1-APP-CX3CR1 ${ }^{+/-}$mice. Expression of IDE (A) MMP9 (B) was analyzed by GPCR on RNA isolated from microglia freshly harvested from brains of PS1-APP, WT littermates, and PS1-APP-CX3CR1+/- mice at 24 months of age: WT brain $(n=6)$, WT microglia $(n=6)$, PS1-APP $(n=10)$, and PS1-APP-CX3CR1 ${ }^{+/-}(n=6)$. Levels of enzymes are expressed relative to the $\beta 2 \mathrm{M}$ housekeeping gene and bars represent mean \pm SEM. * represent statistically significant.

of $A \beta$ degrading enzymes in microglia, and that the preservation of MMP9 observed in mouse brains was due to the effects of CX3CR1-deficiency on cells other than microglia.

\section{CX3CR1 Deficiency Is Associated With Improved Barnes Maze Function in PS1-APP-CX3CR1+/- Mice}

To determine if the observed reduction in brain $A \beta$ levels is associated with improved cognitive and memory functions in PS1-APP-CX3CR1 $1^{+/-}$deficient mice, we tested visuo-spatial learning and memory in these mice using the Barnes maze. PS1-APP, PS1-APP/CX3CR $1^{+/-}$, and WT littermate mice were tested at 300 days of age as detailed in materials and methods. Figure 7A shows results of escape latency (time to find target and hide in it) for the non-cued hidden fixed arm of the study on the four training days. On Day 1 there were no significant differences in the escape latencies between PS1-APP, PS1-APP$\mathrm{CX} 3 \mathrm{CR} 1^{+/-}$, and WT mice. However, by Day 4 , while all mice groups showed improvement in the escape latency, there were statistically significance decreases in both WT $(15.57 \pm 1.66$, $p<0.05)$ and PS1-APP-CX3CR1 $1^{+/-}$mice (16.27 $\pm 2.06, p<$ $0.05)$ escape latency compared to PS1-APP mice (34.9 $\pm 7.13 \mathrm{~s})$. Figure 7B shows representative Day 4 tracings from mice in all three groups. WT and PS1-APP-CX3CR $1^{+/-}$mice navigated to the escape box quickly, while the PS1-APP mice tended to meander and hesitated longer before identifying the correct hole with the escape box. A similar trend was observed when the mice were tested in the T maze, another test for spatial learning, but the differences did not reach statistical significance (data not shown). To determine if functions other than visuo-spatial learning and memory were different between the three groups, we used the open field test which measures exploratory behavior independent of memory or learning. As expected (27), PS1-APP, PS1-APP$\mathrm{CX} 3 \mathrm{CR} 1^{+/-}$, and WT littermate mice showed no difference in exploratory behavior (data not shown).

\section{DISCUSSION}

This manuscript highlights three important findings. First, we show that partial CX3CR1 deficiency in PS1-APP-CX3CR1 $1^{+/-}$ mice is associated with significant reductions in the amount of $\mathrm{A} \beta$ (1-42) and (1-40) and the number of visible $\mathrm{A} \beta$ deposits in their brains compared to regular PS1-APP mice. Complete deletion of CX3CR1 in models of amyloid deposition have been shown to reduce $\mathrm{A} \beta$ deposits and enhanced microglial $\mathrm{A} \beta$ phagocytosis $(16,17)$. However, such complete deletion has also been associated with worsening memory deficits and tau pathology (28-31). Our findings show that partial CX3CR1 deletion restores the beneficial effects of lowering $A \beta$ levels and show improvement in behavioral/cognitive testing. It remains to be seen whether partial CX3CR1 deletion affects tau pathology. However, in combined models of $A \beta$ and tau pathology, $A \beta$ deposition precedes and seems to drive tau pathology. We 
A

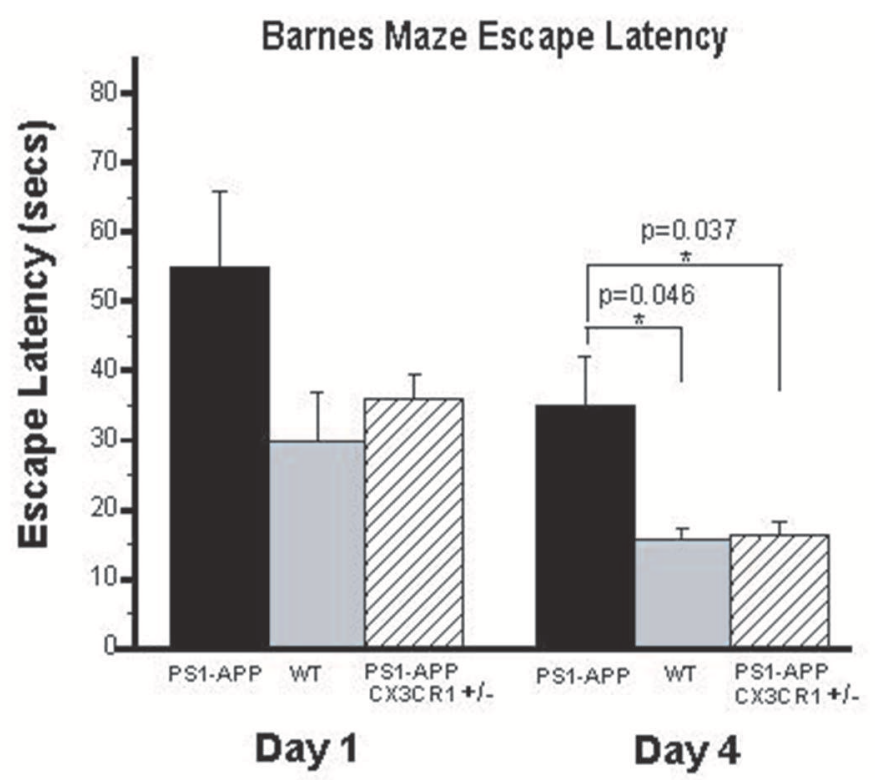

B

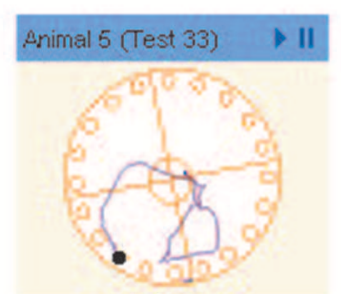

PS1-APP

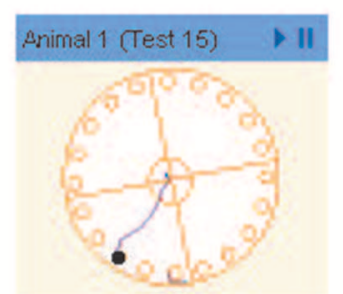

wT

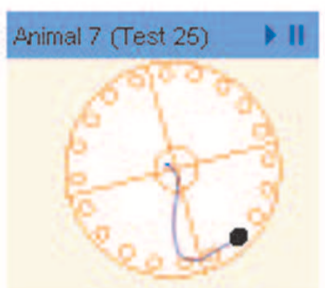

PS1-APP / CXЗCR1 +/-

Day 4 Tracings of Barnes Maze

FIGURE 7 | PS1-APP-CX3CR1 1/- mice perform better that PS1-APP in the Barnes maze behavioral test. Mice at 10-months of age were first trained for 5 days on cued-target Barnes maze and then data collected during a 4-day training using hidden-fixed target protocol on Barnes maze. Escape latency, which is the time for a subject to locate and enter the escape box, was collected for each mouse during all trials and is shown in (A) for Days 1 and 4 of the hidden-fixed target Barnes maze protocol (mean escape latency in $\mathrm{S} \pm$ SEM). On Day 1 there were no significant differences in escape latencies between genotypes. By Day 4, PS1-APP animals took significantly longer to escape the maze compared with both WT and PS1-APP-CX3CR1 $1^{+/-}$mice. (B) Shows representative maze tracings for each genotype on Day 4 of the hidden-fixed target protocol. Animal numbers are WT $(n=6)$, PS1-APP $(n=8)$ and PS1-APP-CX3CR1 ${ }^{+/-}(n=6)$. ${ }^{*}$ represent statistically significant.

are currently exploring the effects of partial CX3CR1 deletion on disease progression in one of these combined models. Importantly, it is unlikely that pharmacologic targeting of CX3CR1 will achieve the effects of complete deletion of this receptor as in $\mathrm{CX} 3 \mathrm{CR} 1^{-/-}$mice. However, it is possible to achieve partial inhibition of CX3CR1 activity pharmacologically, similar to what is observed in $\mathrm{CX} 3 \mathrm{CR} 1^{+/-}$mice heterozygous for this receptor suggesting that the beneficial effects of partial blockade of CX3CR1 function are preserved while mitigating the harmful effects of such blockade.

The second important finding is that partial CX3CR1 deficiency is associated with altered gene expression of neuronal $\mathrm{A} \beta$ degrading enzymes. Microglia do not exist statically in the brain but actively patrol their immediate environment by constant extension and retraction of dendritic-like arms (31). During the course of their normal functioning they make contact with the surfaces of other cells, including neurons. Given the paired expression of CX3CL1 (fractalkine) on neurons and CX3CR1 on microglia in the brain, it has been proposed that the interactions between neurons and microglia mediated through the fractalkine-CX3CR1 complex may modulate microglia functions (30). Our data suggest that a reciprocal interaction may also be occurring and that microglia may regulate neuronal function and gene expression profile via the fractalkine-CX3CR1 pathway. Indeed, by examining the gene expression profile of whole brains and of purified microglia, we found that partial microglial CX3CR1 deficiency reverses or slows the reduction in insulysin and MMP9 
A $\beta$-degrading enzymes observed in PS1-APP mice as they age, and their AD-like pathology progresses. For insulysin, such restoration appears to occur exclusively in neurons, since IDE is expressed predominantly in neurons as evidenced by immuofluorescence staining of brain slices. Similarly, when we evaluated the $A \beta$-degrading enzyme MMP9, which is expressed in microglia and neurons, we also found reduced expression with aging and $\mathrm{AD}$ progression, CX3CR1 deficiency did not restore expression of this enzyme to WT levels in microglia but there was restoration of MMP9 in whole brain. These observations are not explained by a decrease in microglial number since the total number of microglia in the brains of PS1-APP and PS1-APP-CX3CR1 ${ }^{+/-}$were similar (not shown). These findings indicate that microglial CX3CR1 regulates expression of neuronal $\mathrm{A} \beta$ degrading enzymes. Because the only known ligand for microglial CX3CR1 is neuronal fractalkine, these findings suggest that interactions of microglial CX3CR1 with neuronal fractalkine regulate gene expression in neurons.

Heterozygous CX3CR $1^{+/-}$mice have been used in lieu of WT mice when studying the effects of complete deletion of CX3CR1 or when using CX3CR1-CRE mice. The third important finding is that our data show that, at least in the case of models of $A \beta$ deposition, CX3CR1 $1^{+/-}$are clearly different that CX3CR1 $1^{+/+}$ mice and that partial CX3CR1 deficiency significantly alters microglial functions.

It is important to note when interpreting our findings is that due to limited availability of mice of different genotypes, these experiments were performed in male mice. It is to be determined if female mice will exhibit similar differences between the genotypes.

Collectively, our data indicate that partial CX3CR1 deficiency slows progression of $\mathrm{AD}$-like pathology in a transgenic mouse

\section{REFERENCES}

1. Thinakaran G, Koo EH. Amyloid precursor protein trafficking, processing, and function. J Biol Chem. (2008) 283:29615-9. doi: 10.1074/jbc.R800019200

2. Leissring MA. The AbetaCs of Abeta-cleaving proteases. J Biol C. (2008) 283:29645-9. doi: 10.1074/jbc.R800022200

3. Miners JS, Baig S, Palmer J, Palmer LE, Kehoe PG, Love S. Abetadegrading enzymes in Alzheimer's disease. Brain Pathol. (2008) 18:240-52. doi: 10.1111/j.1750-3639.2008.00132.x

4. El Khoury J, Hickman SE. Mechanism of Amyloid Beta clearance in Alzheimer's disease in Research Progress in Alzheimer's Disease and Dementia (Vol 4). New York, NY: Nova Science Publishers Inc. (2009).

5. Hickman SE, Allison EK, El Khoury J. Microglial dysfunction and defective beta-amyloid clearance pathways in aging Alzheimer's disease mice. J Neurosci. (2008) 28:8354-60. doi: 10.1523/JNEUROSCI. 0616-08.2008

6. Hickman SE, El Khoury J. The neuroimmune system in Alzheimer's disease: the glass is half full. J Alzheimers Dis. (2013) 33(Suppl 1):S295-302. doi: 10.3233/JAD-2012-129027

7. Bazan JF, Bacon KB, Hardiman G, Wang W, Soo K, Rossi D, et al. A new class of membrane-bound chemokine with a CX3C motif. Nature. (1997) 385:640-4. doi: 10.1038/385640a0

8. Hickman SE, El Khoury J. Mechanisms of mononuclear phagocyte recruitment in Alzheimer's disease. CNS Neurol Disord Drug Targets. (2010) 9:168-73. doi: 10.2174/187152710791011982 model of the disease and reverses cognitive deficit, by restoring neuronal $\mathrm{A} \beta$-degrading pathways possibly by disrupting CX3CR1-fractalkine interactions. Partial down-regulation of microglial CX3CR1 expression, and/or inhibition CX3CR1fractalkine interactions should be explored as a potential therapeutic target to delay disease progression and improve cognition in $\mathrm{AD}$.

\section{DATA AVAILABILITY STATEMENT}

The datasets generated for this study are available on request to the corresponding author.

\section{ETHICS STATEMENT}

All protocols were approved by the Massachusetts General Hospital Institutional Animal Care and Use Committee and met US National Institutes of Health guidelines for the humane care of animals.

\section{AUTHOR CONTRIBUTIONS}

$\mathrm{SH}$ designed the study, performed and co-analyzed the experiments, and co-wrote the manuscript. EA, UC, and NK-G performed experiments and co-analyzed data. JE conceived the study, designed the experiments, co-analyzed the data, and co-wrote the manuscript.

\section{FUNDING}

This work was supported by NIH grant RF1 AG051506 to JE and a grant from the Dana Foundation Neuroimmunology Program to JE.
9. Hickman SE, Kingery ND, Ohsumi TK, Borowsky ML, Wang LC, Means TK, et al. The microglial sensome revealed by direct RNA sequencing. Nat Neurosci. (2013) 16:1896-905. doi: 10.1038/nn.3554

10. Hughes PM, Botham MS, Frentzel S, Mir A, Perry VH. Expression of fractalkine (CX3CL1) and its receptor, CX3CR1, during acute and chronic inflammation in the rodent CNS. Glia. (2002) 37:314-27. doi: 10.1002/glia.10037

11. D'Haese JG, Demir IE, Friess H, Ceyhan GO. Fractalkine/CX3CR1: why a single chemokine-receptor duo bears a major and unique therapeutic potential. Expert Opin Ther Targets. (2010) 14:207-19. doi: 10.1517/ 14728220903540265

12. Combadiere C, Potteaux S, Gao JL, Esposito B, Casanova S, Lee EJ, et al. Decreased atherosclerotic lesion formation in CX3CR1/apolipoprotein E double knockout mice. Circulation. (2003) 107:1009-16. doi: 10.1161/01.CIR.0000057548.68243.42

13. Lesnik P, Haskell CA, Charo IF. Decreased atherosclerosis in CX3CR $1^{-/-}$ mice reveals a role for fractalkine in atherogenesis. J Clin Invest. (2003) 111:333-40. doi: 10.1172/JCI15555

14. Zhuang ZY, Kawasaki Y, Tan PH, Wen YR, Huang J, Ji RR. Role of the CX3CR1/p38 MAPK pathway in spinal microglia for the development of neuropathic pain following nerve injury-induced cleavage of fractalkine. Brain Behav Immun. (2007) 21:642-51. doi: 10.1016/j.bbi.2006.11.003

15. Rimaniol AC, Till SJ, Garcia G, Capel F, Godot V, Balabanian K, et al. The CX3C chemokine fractalkine in allergic asthma and rhinitis. J Allergy Clin Immunol. (2003) 112:1139-46. doi: 10.1016/j.jaci.2003.09.041 
16. Lee S, Varvel NH, Konerth ME, Xu G, Cardona AE, Ransohoff RM, et al. CX3CR1 deficiency alters microglial activation and reduces beta-amyloid deposition in two Alzheimer's disease mouse models. Am J Pathol. (2010) 177:2549-62. doi: 10.2353/ajpath.2010.100265

17. Liu Z, Condello C, Schain A, Harb R, Grutzendler J. CX3CR1 in microglia regulates brain amyloid deposition through selective protofibrillar amyloid-beta phagocytosis. J Neurosci. (2010) 30:17091-101. doi: 10.1523/JNEUROSCI.4403-10.2010

18. Borchelt DR, Ratovitski T, van Lare J, Lee MK, Gonzales V, Jenkins NA, et al. Accelerated amyloid deposition in the brains of transgenic mice coexpressing mutant presenilin 1 and amyloid precursor proteins. Neuron. (1997) 19:93945. doi: 10.1016/S0896-6273(00)80974-5

19. Jankowsky JL, Slunt HH, Ratovitski T, Jenkins NA, Copeland NG, Borchelt DR. Co-expression of multiple transgenes in mouse CNS: a comparison of strategies. Biomol Eng. (2001) 17:157-65. doi: 10.1016/S1389-0344(01) 00067-3

20. Jung S, Aliberti J, Graemmel P, Sunshine MJ, Kreutzberg GW, Sher A, et al. Analysis of fractalkine receptor CX(3)CR1 function by targeted deletion and green fluorescent protein reporter gene insertion. Mol Cell Biol. (2000) 20:4106-14. doi: 10.1128/MCB.20.11.4106-4114.2000

21. El Khoury JB, Moore KJ, Means TK, Leung J, Terada K, Toft M, et al. CD36 mediates the innate host response to beta-amyloid. J Exp Med. (2003) 197:1657-66. doi: 10.1084/jem.20021546

22. El Khoury J, Toft M, Hickman SE, Means TK, Terada K, Geula C, et al. Ccr2 deficiency impairs microglial accumulation and accelerates progression of Alzheimer-like disease. Nat Med. (2007) 13:432-8. doi: 10.1038/nm1555

23. Reiserer RS, Harrison FE, Syverud DC, McDonald MP. Impaired spatial learning in the APPSwe + PSEN1DeltaE9 bigenic mouse model of Alzheimer's disease. Genes Brain Behav. (2007) 6:54-65. doi: 10.1111/j.1601-183X.2006.00221.X

24. Miller BC, Eckman EA, Sambamurti K, Dobbs N, Chow KM, Eckman CB, et al. Amyloid-beta peptide levels in brain are inversely correlated with insulysin activity levels in vivo. Proc Natl Acad Sci USA. (2003) 100:6221-6. doi: 10.1073/pnas.1031520100

25. Paglinawan R, Malipiero U, Schlapbach R, Frei K, Reith W, Fontana A. TGFbeta directs gene expression of activated microglia to an anti- inflammatory phenotype strongly focusing on chemokine genes and cell migratory genes. Glia. (2003) 44:219-31. doi: 10.1002/glia.10286

26. Maolood N, Hardin-Pouzet H, Grange-Messent V. Matrix metalloproteinases MMP2 and MMP9 are upregulated by noradrenaline in the mouse neuroendocrine hypothalamus. Eur J Neurosci. (2008) 27:1143-52. doi: 10.1111/j.1460-9568.2008.06099.x

27. Lalonde R, Kim HD, Fukuchi K. Exploratory activity, anxiety, and motor coordination in bigenic APPswe + PS1/DeltaE9 mice. Neurosci Lett. (2004) 369:156-61. doi: 10.1016/j.neulet.2004.07.069

28. Bhaskar K, Konerth M, Kokiko-Cochran ON, Cardona A, Ransohoff RM, Lamb BT. Regulation of tau pathology by the microglial fractalkine receptor. Neuron. (2010) 68:19-31. doi: 10.1016/j.neuron.2010. 08.023

29. Cho SH, Sun B, Zhou Y, Kauppinen TM, Halabisky B, Wes P, et al. CX3CR1 protein signaling modulates microglial activation and protects against plaqueindependent cognitive deficits in a mouse model of Alzheimer disease. J Biol Chem. (2011) 286:32713-22. doi: 10.1074/jbc.M111.254268

30. Guedes JR, Lao T, Cardoso AL, El Khoury J. Roles of microglial and monocyte chemokines and their receptors in regulating Alzheimer's diseaseassociated amyloid-beta and tau pathologies. Front Neurol. (2018) 9:549. doi: 10.3389/fneur.2018.00549

31. Hickman S, Izzy S, Sen P, Morsett L, El Khoury J. Microglia in neurodegeneration. Nat Neurosci. (2018) 21:1359-69. doi: $10.1038 / \mathrm{s} 41593-018-0242-\mathrm{x}$

Conflict of Interest: The authors declare that the research was conducted in the absence of any commercial or financial relationships that could be construed as a potential conflict of interest.

Copyright (C) 2019 Hickman, Allison, Coleman, Kingery-Gallagher and El Khoury. This is an open-access article distributed under the terms of the Creative Commons Attribution License (CC BY). The use, distribution or reproduction in other forums is permitted, provided the original author(s) and the copyright owner(s) are credited and that the original publication in this journal is cited, in accordance with accepted academic practice. No use, distribution or reproduction is permitted which does not comply with these terms. 